\title{
Azoles de antes y ahora: una revisión
}

\author{
Azoles of then and now: a review
}

\author{
Laura Cristina Nocua-Báez', Paula Uribe-Jerez', Leonardo Tarazona-Guaranga1, Ricardo Robles y Jorge A. Cortés
}

${ }^{1}$ Departamento de Medicina Interna, Facultad de Medicina, Universidad Nacional de Colombia, Bogotá, Colombia.

Financiamiento: No hay una fuente de financiación específica para este manuscrito.

Conflictos de interés: JAC ha recibido financiación de proyectos de investigación de Pfizer y Merck Sharp and Dohme.

Los otros autores no tienen conflictos de interés que declarar (LCNB, PUJ, LTG, RR).

Recibido (nueva versión): 23 de diciembre de 2019 / Aceptado: 9 de marzo de 2020

\section{Resumen}

Los azoles son fármacos que inhiben la enzima $14 \alpha$-esteroldemetilasa, impidiendo la unión de ergosterol; esto altera la estructura y función de la pared celular fúngica. Especialmente el grupo de los triazoles: fluconazol, itraconazol, voriconazol, posaconazol e isavuconazol, son una alternativa farmacológica para el tratamiento de la enfermedad fúngica invasora causada por Aspergillus spp, Candida spp, Cryptococcus spp, patógenos emergentes como los Mucorales, y de micosis endémicas como las ocasionadas por Histoplasma spp y Coccidioides spp. Los efectos adversos de los triazoles son menos frecuentes comparados con los ocasionados por anfotericina $\mathrm{B}$, un antifúngico de uso común para estas micosis. Los principales efectos adversos de los triazoles son hepáticos, gastrointestinales y cardiovasculares como la prolongación del intervalo QT. Las interacciones farmacológicas son usuales y se presentan con moléculas que usan sustratos del citocromo CYP3A4, lo que incluye anti-retrovirales, anti-tuberculosos e inmunomoduladores. En este trabajo se revisan la historia, características farmacológicas y los ensayos clínicos que evidencian su eficacia clínica en los diferentes escenarios clínicos.

Palabras clave: azoles/administración y dosificación; azoles/uso terapéutico; azoles/farmacología; azoles/efectos adversos; candidiasis; candidiasis/terapia; aspergilosis; aspergilosis/terapia; criptococosis; criptococosis/terapia; histoplasmosis; histoplasmosis/terapia; mucormicosis; mucormicosis/terapia.

\section{Introducción}

S e ha observado un incremento en la incidencia de las micosis invasoras, a pesar del advenimiento de nuevos antifúngicos, debido a la invención de nuevos tratamientos para patologías

\begin{abstract}
The azoles are drugs that inhibit the $14 \alpha$-sterol-demethylase enzyme preventing the binding of ergosterol, altering the functionality and structure of the fungal cell wall. Especially the group of triazoles: fluconazole, itraconazole, voriconazole, posaconazole and isavuconazole, are a pharmacological alternative for the treatment of the invasive fungal disease, caused by Aspergillus spp, Candida spp, Cryptococcus spp, by emerging pathogens for example, the Mucoral and finally of endemic mycosis as those caused by Histoplasma spp. and Coccidioides spp. The adverse effects of the triazoles are less frequent compared to those caused by amphotericin B, the main ones being hepatics, gastrointestinals and cardiovasculars, such as the prolongation of the QT interval. The pharmacological interactions are common and occur with molecules that use the substrates of the CYP3A4 cytochrome, for example: antiretroviral, anti-tuberculous and immunomodulators. The history, pharmacological characteristics and clinical trials are reviewed.

Keywords: azoles/administration and dosage; azoles/therapeutic use; azoles/pharmacology; azoles/adverse events; candidiasis; candidiasis/therapy; aspergillosis; aspergillosis/therapy; cryptococosis; cryptococosis/therapy; histoplasmosis; histoplasmosis/therapy; mucormycosis; mucormycosis/therapy.
\end{abstract}

\section{Correspondencia a:}

Jorge Alberto Cortés Luna

jacortesl@unal.edu.co

crónicas que pueden afectar el sistema inmunológico, lo que predispone a sufrir estas infecciones.

Las enfermedades fúngicas invasoras (EFI), tienen una alta mortalidad, llegando a ser mayor a 50\% en el caso de la aspergilosis invasora (AI) y de $30 \%$ en la candidemia ${ }^{1}$. Los factores de riesgo descritos 
para EFI son: la inmunosupresión, la quimioterapia por enfermedades neoplásicas (especialmente con neutropenia para AI), los individuos receptores de trasplante de progenitores hematopoyéticos (TPH) o de órganos sólidos (TOS), quienes tienen diagnóstico de infección por virus de la inmunodeficiencia humana (VIH) con bajo conteo de linfocitos T CD4, los pacientes que reciben dosis prolongadas de corticosteroides u otros inmunosupresores, aquellos en post-operatorio de cirugía mayor, y ancianos, condiciones que son comunes en una población cuya esperanza de vida va en aumento ${ }^{2,3}$.

Todos los tejidos, órganos y líquidos orgánicos estériles pueden afectarse por una EFI, y el grupo de los microorganismos patógenos causales está en ascenso. Los más frecuentemente descritos son las especies de Candida y Aspergillus, Cryptococcus neoformans y Pneumocystis jirovecii. Candida es la principal responsable de EFI, con una frecuencia variable según la enfermedad de base del paciente; en el caso de receptores de TPH de 31,6\%, 92\% en pacientes en post-operatorio de cirugía mayor y $89 \%$ en pacientes con neoplasias ${ }^{4}$.

En América Latina, la especie de Candida más común es $C$. albicans en $37,6 \%$ de los casos, seguida por $C$. parapsilopsis $26,5 \%$, documentándose en los últimos años una elevación de la proporción de eventos por Candida no albicans (C. parapsilosis, C. tropicalis y C. krusei) superior a $50 \%{ }^{2}$.

En los pacientes con neoplasias hematológicas y trasplante de células madre hematopoyéticas (TCMH), Aspergillus spp, es la etiología más frecuente de EFI y $A$. fumigatus es la especie predominante ${ }^{5}$. Las micosis invasoras endémicas son características de algunos entornos geográficos, y los patógenos descritos son: Histoplasma capsulatum, Coccidioides immitis, Blastomyces dermatitidis o Paracoccidioides brasiliensis ${ }^{1}$. También son importantes los hongos del orden Mucorales de la clase de Zigomicetos, que incluyen: Rhizopus spp., Mucor spp., Rhizomucor, y Leichtheimia spp, patógenos emergentes que se han aislado con mayor frecuencia en EFI en los últimos años, con una frecuencia de hasta 5,2\% en neoplasias hematológicas ${ }^{3,6}$.

Inicialmente se disponía de anfotericina B para el tratamiento de las micosis invasoras, pero su nefrotoxicidad, reacciones a la infusión y trastornos electrolíticos, llevaron al desarrollo de los azoles como alternativa más segura. Los azoles se dividen en imidazoles, que son limitados para micosis superficiales: ketoconazol, miconazol, clotrimazol, y el segundo grupo es el de triazoles, con un amplio rango de aplicaciones: fluconazol, itraconazol, voriconazol, posaconazol e isavuconazol ${ }^{3}$. Preocupante es el surgimiento de resistencia a azoles; en la parte norte de Sudamérica se han identificado aislados de Candida auris con resistencia importante a azoles, mientras que $A$. fumigatus es intrínsecamente resistente a fluconazol (por codificación en el gen CYP51A) ${ }^{7,8}$. El objetivo de esta revisión es describir las características farmacológicas de los azoles, su espectro de acción microbiológica, la aplicación clínica y las limitaciones de su utilización.

\section{Historia}

En 1969 fueron comercializados los primeros azoles: clotrimazol y miconazol como alternativas a griseofulvina y nistatina para infecciones cutáneas y muco-cutáneas causadas por dermatofitos y Candida spp. (Figura 1).

Diez años después fue aprobado el uso intravenoso de miconazol, pero presentó efectos adversos, gastrointestinales, fiebre, rash cutáneo, muy frecuentes, por lo que su uso fue suspendido ${ }^{9,10}$.

En 1981 fue aprobado ketoconazol oral como alternativa a anfotericina $\mathrm{B}$, pero su relación con hepatotoxicidad intensa y disminución de los niveles de testosterona y cortisol, hizo que esta vía de administración fuera restringida, y en 2013 la Food and Drug Administration (FDA por sus siglas en inglés) indicó usarla solamente cuando otros antifúngicos no fueran tolerados o no estuvieran disponibles. Además, la comisión europea en este mismo año ratificó la orden de suspender la comercialización de la forma de administración oral de ketoconazol $\left(\text { Nizoral }^{\circledR}\right)^{11,12}$. En la actualidad se dispone del champú tópico, crema, y gel ${ }^{11,13}$. En los países de Latinoamérica también fue retirado el ketoconazol oral; fue así como el 2 de enero de 2014, en Panamá, el Ministerio de Salud con la resolución $\mathrm{N}^{\circ} 516$ del 06 de diciembre de 2013, ordenó cancelar los registros sanitarios de los medicamentos orales y de acción sistémica que contuvieran como principio activo ketoconazol ${ }^{14}$.

En 1990 surgió fluconazol oral e intravenoso, un triazol con perfil de seguridad y aplicación clínica mejor que ketoconazol, incluyendo la acción del fluconazol contra Candida spp, C. neoformans y hongos dimórficos ${ }^{15}$.

Dos años después, en 1992, se inició el uso de itraconazol oral en cápsulas y en 1997 en solución, con espectro para Aspergillus spp, histoplasmosis y blastomicosis, con la aparición de efectos gastrointestinales y el fenómeno de absorción dependiente de los alimentos y el pH gástrico similar a ketoconazol; en 1999 se comercializó una preparación intravenosa que rápidamente fue retirada del mercado por una alta incidencia de efectos adversos ${ }^{3}$.

Debido al cambio de los patógenos responsables de EFI, con un predominio de $C$. no albicans, y el aumento en la incidencia de hongos emergentes refractarios como Scedosporium spp. y Fusarium spp., surgieron otros triazoles, como voriconazol oral e intravenoso en el año 2002, aunque algunas de las especies consideradas en este grupo son resistentes ${ }^{16,17}$. 
En el año 2006, fue aprobado posaconazol oral en suspensión, con un espectro adicional a voriconazol, que incluye los mucorales; en 2013 se inició la comercialización de las tabletas y en 2014 de la presentación intravenosa ${ }^{3}$. Los cambios en las presentaciones farmacéuticas de posaconazol favorecieron su administración y con la introducción de tabletas se superaron ciertas limitaciones para absorción del fármaco en suspensión, dado que ésta era baja.

El más reciente triazol aprobado por la FDA es isavuconazol como alternativa para el tratamiento de la AI y la mucormicosis ${ }^{18}$ (Figura 1).

\section{Química}

Los azoles conforman un grupo heterogéneo de antifúngicos que comparten la característica de un anillo imidazólico libre unido mediante enlace carbononitrógeno a otros anillos aromáticos, según los cuales hay cambios en las propiedades físico-químicas, los efectos terapéuticos y la toxicidad de los fármacos. De acuerdo al número de los nitrógenos que tenga el anillo, se dividen a los azoles en dos grupos: el primero es de los triazoles, que tienen tres nitrógenos en el anillo principal e incluye a fluconazol, itraconazol, voriconazol que tiene un grupo de fluoro pirimidina como única diferencia, posaconazol e isavuconazol; el segundo grupo es el de los imidazoles, que tienen dos nitrógenos en la estructura del anillo, y corresponden a miconazol, ketoconazol y clotrimazol ${ }^{19,20}$ (Figura 2).

\section{Mecanismo de acción}

La pared celular de los hongos le confiere su propiedad de plasticidad, es el control de la permeabilidad y la armadura que lo defiende de los cambios osmóticos. La integridad de esta pared depende de los esteroles que la conforman, como el ergosterol, componente lipídico principal, que favorece la acción de la quitina sintetasa, encargada del crecimiento y división celular fúngica. Los azoles triazoles inhiben la $14 \alpha$-esterol-demetilasa dependiente del citocromo P-450, cuya función es unir los precursores del ergosterol, conduciendo al acúmulo de estas moléculas precursoras que incluyen los $14 \alpha$-metilados (lanosterol, 4,14-dimetil-zimosterol y 24-metilen-dihidro-

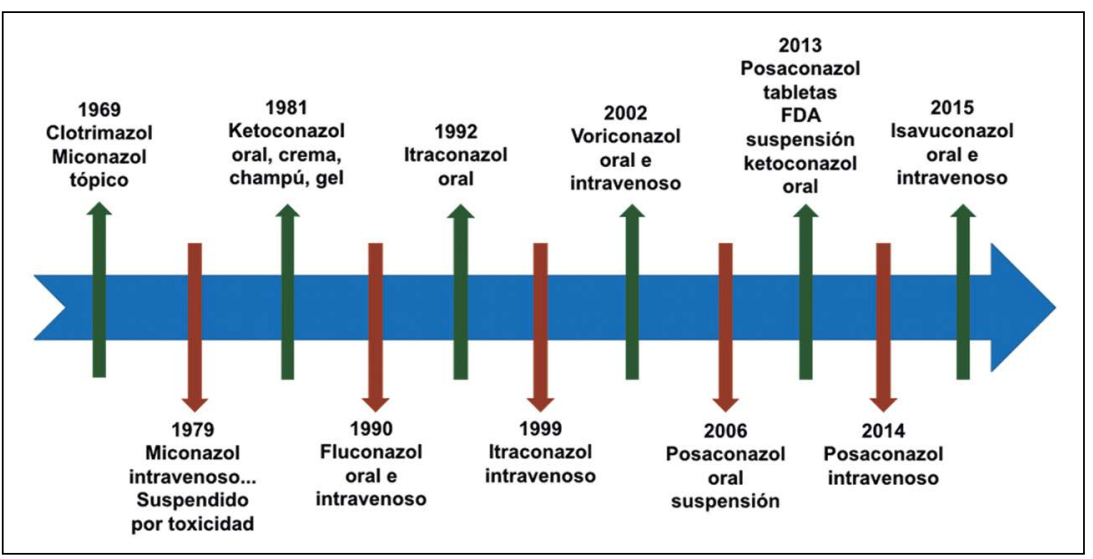

Figura 1. Historia de aparición de los azoles.

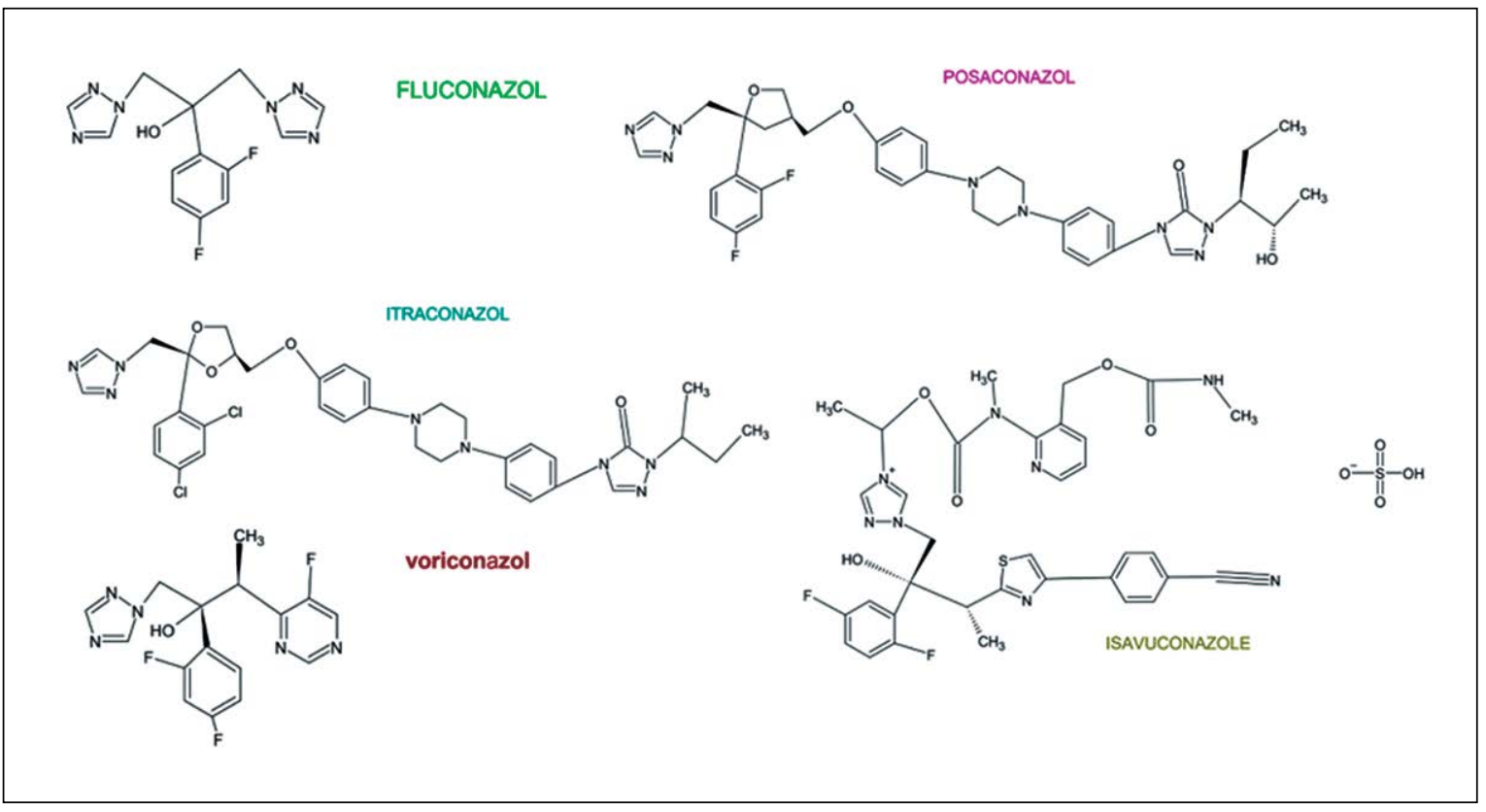

Rev Chilena Infectol 2020; 37 (3): 219-230
Figura 2. Estructura química de fluconazol, itraconazol, voriconazol, posaconazol e isavuconazol. 


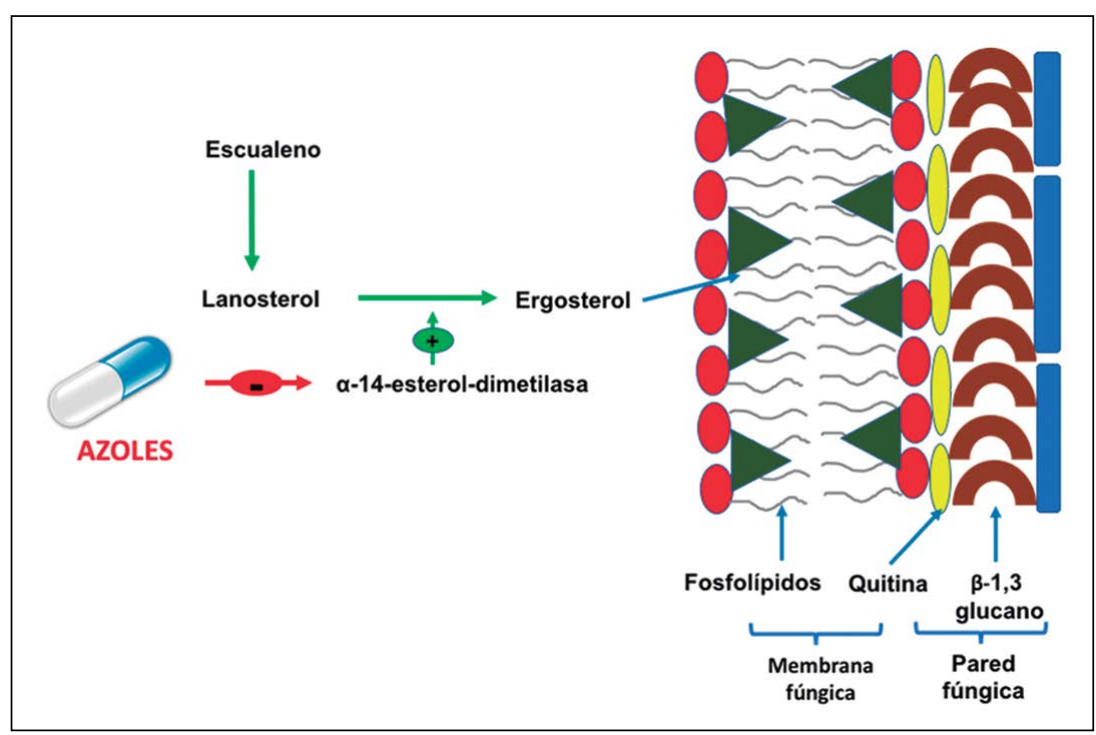

Figura 3. Mecanismo de acción de los azoles. la bomba de protones pueden disminuir la absorción de algunos azoles). Las concentraciones plasmáticas de estos medicamentos suelen ser bajas pero tienen un buen volumen de distribución lo que favorece el efecto deseado ${ }^{25}$.

La vía de eliminación de los azoles es principalmente hepática, seguida por la renal y biliar. Los triazoles presentan un comportamiento farmacológico que depende de la concentración y el tiempo de exposición; además, poseen un efecto post-antifúngico prolongado. El principal parámetro farmacocinético/farmacodinámico $[\mathrm{PK} / \mathrm{PD}]$ que define la eficacia de estos agentes es la exposición total a medicamento (área bajo la curva 0-24h/concentración inhibitoria mínima $[\mathrm{ABC} / \mathrm{CIM}])^{26}$. El objetivo terapéutico se puede lograr con relaciones ABC/CIM (basados en la concentración libre del medicamento) entre 11 y 100 , dependiendo del medicamento y microorganismo a cubrir.

La característica lipofílica de los azoles, hace que sea necesario combinarlos con moléculas como la ciclodextrina, que se convierte en el vehículo de transporte del fármaco; se ha demostrado que este compuesto en pacientes con enfermedad renal y tasa de filtración glomerular menor a $50 \mathrm{ml} / \mathrm{min} / 1,73 \mathrm{~m}^{2}$, se acumula, precipitando la ocurrencia de más efectos adversos relacionados con la nefrotoxicidad; sin embargo, su impacto clínico sigue sin estar claro. Es así como en pacientes adultos con enfermedad renal crónica y neoplasias hematológicas, a quienes se administró voriconazol endovenoso para el tratamiento de AI, no se encontró deterioro de la función renal ni efectos adversos graves ${ }^{27}$.

Algunas características específicas de estos azoles son:

\section{Fluconazol}

Fluconazol es soluble en agua y muy bien absorbido en el tracto gastrointestinal, en más de $90 \%$, su absorción no se ve afectada por el $\mathrm{pH}$ o la ingesta de los alimentos y tiene mínima metabolización hepática. Difunde en líquidos corporales incluyendo el líquido cefalorraquídeo (LCR) donde alcanza una concentración de hasta 70\% de la concentración sérica total ${ }^{20}$. Tiene una gran eliminación renal, por lo cual en pacientes con falla renal la dosis debe ser reducida a $50 \%$ y en el caso de pacientes que se encuentren en terapia de reemplazo renal, como la hemodiálisis, una dosis completa debe ser administrada después de cada sesión. Este medicamento tiene un volumen de distribución bajo por lo que sus concentraciones séricas son mayores ${ }^{25,28}$.

Los azoles tienen diferentes características farmacocinéticas y farmacodinámicas. En general, su absorción oral es buena, mayor a 50\%; incluso, en el caso de voriconazol llega a ser cercana a $100 \%$, pero son muchos los factores endógenos que influyen en su absorción, como la ingestión del medicamento en presencia de alimentos especialmente ricos en grasa, y factores que afecten el $\mathrm{pH}$ de la mucosa gástrica (los medicamentos inhibidores de

\section{Itraconazol y posaconazol}

Itraconazol y posaconazol comparten varias de sus características farmacológicas; son pobremente solubles en agua por lo que requieren ser administrados con ciclodextrina para mejorar su solubilidad, la cual puede ser potencialmente tóxica; entre estos tres azoles, el agente más lipofílico es itraconazol. La absorción de todos los 
azoles se ve alterada en gran medida por la composición de los alimentos ingeridos; la absorción de posaconazol se ve favorecida cuando se ingieren con alimentos ricos en grasas ${ }^{29}$. La ciclodextrina también se ha utilizado para favorecer la solubilidad del itraconazol oral en solución y mejorar su biodisponibilidad en comparación con las cápsulas $^{30}$, fenómeno que tiene mayor beneficio en los pacientes inmunosuprimidos. Inicialmente, la forma de presentación de posaconazol fue la suspensión oral, pero debido a la limitación de su absorción saturable y la alta variabilidad de su bio-disponibilidad, dependiendo de la presencia de grasas e incluso de bebidas gaseosas ácidas, se decidió dar paso a otras formas de presentación del medicamento, como las tabletas de liberación retardada, con las cuales se logró reducir la liberación activa del fármaco en condiciones de un $\mathrm{pH}$ gástrico bajo e incrementarla a un $\mathrm{pH}$ elevado del intestino. Con esta forma de presentación del fármaco se consiguió una bio-disponibilidad de $54 \%{ }^{31}$.

Itraconazol y posaconazol, tienen volúmenes de distribución elevados y muy variables, lo cual hace difícil determinar sus concentraciones séricas. Ambos son ampliamente metabolizados en el hígado y pueden generar múltiples interacciones farmacológicas con medicamentos que comparten la vía metabólica de la CYP450. Tienen una eliminación renal muy variable, por lo que no dependen de la función de este sistema.

La gran variabilidad de sus características farmacológicas los convierten en medicamentos menos predecibles y ligeramente más complejos de manejar ${ }^{20,25}$.

\section{Voriconazol}

Voriconazol es pobremente soluble en agua, por lo que debe ser administrado con ciclodextrina; este medicamento tiene el mejor perfil de absorción oral, respecto a los demás azoles, cercana a $100 \%$ en ayunas ${ }^{29}$, el tiempo medio hasta la concentración plasmática máxima varía entre 1,43 h para el caso de la dosis de $200 \mathrm{mg}$ y $1,81 \mathrm{~h}$ para la de $400 \mathrm{mg}$. Tiene un buen volumen de distribución, pero es menor al descrito para itraconazol, posaconazol e isavuconazol y logra penetrar el sistema nervioso central. Su metabolismo es principalmente hepático y la eliminación es por vía renal; aproximadamente $98 \%$ de sus metabolitos se excretan en el plazo de $48 \mathrm{~h}^{32}$.

\section{Isavuconazol}

Isavuconazol es un potente triazol de segunda generación de reciente introducción, que se administra como pro-fármaco (sulfato de isavuconazonio), que por esterasas del plasma se descompone en la molécula activa: isavuconazol y en un producto de escisión inactivo. Tiene una alta solubilidad en agua por lo que no debe combinarse con la ciclodextrina en la formulación intravenosa para facilitar la solubilidad; este detalle reduce de manera importante la nefrotoxicidad que se ha descrito en algunos trabajos a causa de la ciclodextrina ${ }^{33}$. Este antifúngico tiene un gran volumen de distribución y se encuentra unido a proteínas en más de $99 \%$, principalmente a la albúmina; su bio-disponibilidad absoluta oral alcanza a $98 \%$, por lo que la misma dosis puede ser utilizada para administración oral e intravenosa y su absorción no es dependiente del $\mathrm{pH}$ gástrico o del consumo de otros alimentos. El pico de concentración del fármaco en la forma oral se encuentra a las 2-3 h, y después de 1 hora en el caso de la administración intravenosa. Una de las ventajas de isavuconazol sobre otros azoles es su vida media marcadamente más prolongada, con un promedio de 100-115 h, describiéndose en algunos trabajos de hasta 130 h. La segunda ventaja más grande del isavuconazol corresponde al muy buen volumen de distribución, alcanzando una gran concentración en tejidos como el hepático, la bilis, glándulas suprarrenales, corteza suprarrenal y tejido adiposo marrón, con las concentraciones más bajas en hueso, lente ocular, ojo, vesículas seminales y bulbo olfatorio $^{34,35}$

En la Tabla 1 se resumen las características farmacológicas de los principales azoles de interés clínico.

\section{Monitoreo de las concentraciones terapéuticas de los azoles}

Con el objetivo de maximizar la probabilidad de un resultado satisfactorio y minimizar el riesgo de toxicidad, se ha considerado que para los azoles se puede hacer la medición de la relación $\mathrm{ABC} / \mathrm{CIM}^{36}$, como determinante independiente de las concentraciones terapéuticas de itraconazol y voriconazol.

En el caso de itraconazol, además se ha propuesto el monitoreo del metabolito hidroxi-itraconazol; las concentraciones mayores a $0,25-0,5 \mu \mathrm{g} / \mathrm{mL}$ de itraconazol y $1 \mu \mathrm{g} / \mathrm{mL}$ del metabolito, son los puntos de corte para una adecuada efectividad en la mayoría de las infecciones micóticas.

Con respecto a las concentraciones óptimas de voriconazol, se ha documentado que concentraciones bajo $0,25 \mu \mathrm{g} / \mathrm{mL}$ están asociados con un resultado sub-óptimo y una concentración mínima de $1 \mu \mathrm{g} / \mathrm{mL}$, con $70 \%$ de probabilidad de resultado exitoso, mientras que una concentración de $6 \mu \mathrm{g} / \mathrm{mL}$ tiene aproximadamente $20 \%$ de probabilidad de toxicidad del sistema nervioso central.

Aún no se dispone de evidencia que soporte recomendar la monitorización rutinaria para posaconazol, no se ha establecido una concentración objetivo. Sin embargo, se ha encontrado evidencia de una tasa progresivamente más alta de respuesta con mayores exposiciones al fármaco $\mathrm{y}$ un pico promedio de concentración de 1,25-1,5 $\mu \mathrm{g} / \mathrm{mL}$ se asociado a una tasa de respuesta de $75 \%$, por lo que su monitoreo está indicado en casos particulares, como 


\begin{tabular}{|c|c|c|c|c|c|}
\hline & Fluconazol & Itraconazol & Voriconazol & Isavuconazol & Posaconazol \\
\hline Forma farmacéutica & $\begin{array}{l}\text { Tabletas } \\
\text { Suspensión } \\
\text { Polvo para dilución }\end{array}$ & $\begin{array}{l}\text { Cápsulas } \\
\text { Solución }\end{array}$ & $\begin{array}{l}\text { Tabletas } \\
\text { Suspensión } \\
\text { Polvo para dilución }\end{array}$ & $\begin{array}{l}\text { Tabletas } \\
\text { Polvo para dilución }\end{array}$ & $\begin{array}{l}\text { Tabletas } \\
\text { Suspensión } \\
\text { Polvo para dilución }\end{array}$ \\
\hline $\begin{array}{l}\text { Dosis diaria de mantenimiento } \\
\text { en adultos }\end{array}$ & 400 mg VO e IV & $200 \mathrm{mg}$ VO e IV & $\begin{array}{l}4 \mathrm{mg} / \mathrm{kg} \text { dos veces } \\
\text { al día }\end{array}$ & 372 mg cada día & 400 mg dos veces al día \\
\hline Vida media (h) & $22-31$ & $5-64$ & $6-24$ & 130 & $15-35$ \\
\hline Metabolismo & Mínimo hepático & Hepático (CYP3A4) & $\begin{array}{l}\text { Hepático (CYP2C19, } \\
\text { CYP2C9, CYP3A4) }\end{array}$ & Hepático (CYP3A4) & $\begin{array}{l}\text { Glucoronidación } \\
\text { (UGT1A4) }\end{array}$ \\
\hline Unión a proteínas & $\sim 12 \%$ & $>99 \%$ & $\sim 58 \%$ & $>99 \%$ & $>99 \%$ \\
\hline Volumen de distribución & $0,56-0,82 \mathrm{~L} / \mathrm{kg}$ & $11 \mathrm{~L}$ & $4-6 \mathrm{~L}$ & $456 \mathrm{~L}$ & $7-25 \mathrm{~L}$ \\
\hline Eliminación & $\begin{array}{l}\text { Renal } 89 \% \text { (sin } \\
\text { cambios) }\end{array}$ & Renal 40\% Biliar 55\% & Principalmente renal & $\begin{array}{l}\text { Principalmente en } \\
\text { heces, renal en }<1 \%\end{array}$ & $\begin{array}{l}\text { Hepática } 76 \% \text { Renal } \\
14 \%\end{array}$ \\
\hline Bio-disponibilidad & $>90 \%$ & $\begin{array}{l}\sim 55 \% \text {, (se optimiza con } \\
\text { la ingesta de alimentos); } \\
90 \% \text { con la solución }\end{array}$ & $\begin{array}{l}>90 \% \text {, (disminuye } \\
\text { hasta } 22 \% \text { con la } \\
\text { ingesta de alimentos) }\end{array}$ & $98 \%$ & $\begin{array}{l}<45 \% \text { (varía con la } \\
\text { ingesta de alimentos) }\end{array}$ \\
\hline Penetración en LCR & Sí & No & Sí & Sí & Intermedia \\
\hline
\end{tabular}

en pacientes con fracaso del tratamiento y sospecha de microorganismos resistentes y de fenómenos de malabsorción $^{37,38}$.

\section{Escenarios clínicos de uso de azoles}

\section{Criptococosis}

El tratamiento con azoles en la criptococosis es de elección en el mantenimiento y de alternativa en terapia combinada de primera línea. Pese a que en el estudio realizado por Day y cols. ${ }^{39}$, no se encontró beneficio en la supervivencia en la terapia de combinación de anfotericina B y fluconazol para el tratamiento de la criptococosis meníngea en pacientes con infección por VIH, la guía de la Asociación de Enfermedades Infecciosas de América (IDSA) la recomienda como terapia alternativa para inducción y consolidación. En terapia de mantenimiento, fluconazol es indicación de primer orden y ha demostrado ser altamente efectivo en prevenir la recurrencia de criptococosis meníngea en pacientes con síndrome de la inmunodeficiencia adquirida $(\text { SIDA) })^{40}$.

\section{Candidiasis}

En candidiasis invasora o candidemia, las equinocandinas continúan siendo superiores en eficacia en comparación a los azoles; sin embargo, en pacientes no neutropénicos, sin exposición previa al uso de azoles y baja incidencia de $C$. no albicans, fluconazol ha demostrado eficacia similar frente a otros tratamientos de segunda línea, por lo que está recomendado como terapia alternativa. En candidiasis esofágica, fluconazol oral continúa siendo la terapia elección; no obstante, recientemente las equinocandinas se han posicionado como una terapia alternativa con igual efectividad y seguridad $^{41}$

\section{Aspergilosis e histoplasmosis invasoras}

Respecto a hongos filamentosos, los triazoles son los agentes preferidos para el tratamiento y la prevención de AI. Voriconazol es la primera opción de tratamiento y hay evidencia de que éste condujo a mejores respuestas y mejoró la supervivencia en comparación al enfoque estándar con anfotericina $\mathrm{B}^{42}$. En pacientes con SIDA e histoplasmosis diseminada leve a modera, itraconazol ha demostrado ser una terapia de inducción segura y efectiva $^{43}$. En pacientes con AI, isavuconazol es una alternativa; fue comparado con voriconazol en un estudio de no inferioridad, llamado "SECURITE" y, dados sus buenos resultados, fue aprobado por la FDA ${ }^{44}$.

\section{Micosis endémicas}

Por último, en infecciones por Paracoccidioides spp, las guías brasileras para el manejo clínico de paracoccidioidomicosis recomiendan el tratamiento 
de formas leves y moderadas de la enfermedad con itraconazol que ha demostrado alta eficacia y seguridad. Itraconazol en comparación con cotrimoxazol demostró superioridad significativa y tasas de curación en menor tiempo ${ }^{45}$. Sin embargo, hasta la fecha no se cuentan con ensayos clínicos de alta calidad que sustenten estas recomendaciones.

\section{Mucormicosis}

El tratamiento de las mucormicosis incluye manejo quirúrgico adicional al tratamiento antimicótico. Entre los antimicóticos que se pueden utilizar se incluyen posaconazol y isavuconazol, con estudios en escenarios de tratamiento empírico, y sobre todo, tratamiento en pacientes refractarios o intolerantes a otros medicamentos $^{36}$.

En la Tabla 2. se resume la eficacia clínica de los azoles en diferentes escenarios clínicos. En ella se recoge información de estudios clínicos individuales o meta-análisis que han permitido identificar su utilidad.

Tabla 2. Estudios clínicos sobre el tratamiento de infecciones fúngicas con azoles

\begin{tabular}{|c|c|c|c|c|}
\hline Escenario & Autor, año, referencia & Azol & Comparador & Recomendación \\
\hline \multicolumn{5}{|l|}{ Criptococosis } \\
\hline $\begin{array}{l}\text { Meníngea: fase de } \\
\text { inducción }\end{array}$ & Day $(2013)^{39}$ & $\begin{array}{l}\text { Fluconazol en conjunto con } \\
\text { anfotericina B E: } 80 \%\end{array}$ & $\begin{array}{l}\text { Anfotericina B en conjunto con } \\
\text { flucitosina } \\
\text { E: } 85 \%\end{array}$ & $\begin{array}{l}\text { Se recomienda en terapia de } \\
\text { mantenimiento en pacientes sin } \\
\text { infección persistente }\end{array}$ \\
\hline $\begin{array}{l}\text { Meníngea: fase de } \\
\text { mantenimiento }\end{array}$ & Bozzette $(1991)^{40}$ & $\begin{array}{l}\text { Fluconazol 100-200 mg/día } \\
\text { E: 100\% }\end{array}$ & $\begin{array}{l}\text { Placebo } \\
\text { E: } 85 \%\end{array}$ & $\begin{array}{l}\text { La terapia de mantenimiento con } \\
\text { fluconazol es altamente efectiva } \\
\text { en prevenir la recurrencia de } \\
\text { criptococosis meníngea en pacientes } \\
\text { con SIDA }\end{array}$ \\
\hline Pulmonar & IDSA ${ }^{46}$ & $\begin{array}{l}\text { Fluconazol } 400 \text { mg/día 6-12 } \\
\text { meses }\end{array}$ & & $\begin{array}{l}\text { Para síntomas leves a moderados, } \\
\text { ausencia de infiltrados pulmonares } \\
\text { difusos, ausencia de inmunosupresión } \\
\text { grave y resultados negativos de una } \\
\text { evaluación diagnóstica de difusión }\end{array}$ \\
\hline
\end{tabular}

\section{Candidiasis}

Esofágica

Reboli $(2004)^{47}$

Villanueva $(2002)^{41}$

Orofaríngea

Pons $(1993)^{4}$

Candidemia en paciente no neutropénico

Reboli $(2007)^{47}$

$\operatorname{Re} x^{49}$

Fluconazol 400 mg/día

E: $70 \%$

Fluconazol 200 mg/día E: $85 \%$

Fluconazol 100 mg/día E: $98 \%$

Fluconazol 400 mg/día E: $56,8 \%$

E: $74 \%$

Anidulafungina $100 \mathrm{mg} / \mathrm{dí}$ E: $97,2 \%$

Caspofungina $50 \mathrm{mg} / \mathrm{día}$

E. $81 \%$

Clotrimazol $10 \mathrm{mg}$ cinco veces al día E: $94 \%$

Anidulafungina $100 \mathrm{mg} / \mathrm{día}$

Anfotericina B $0,7 \mathrm{mg} / \mathrm{kg} / \mathrm{día}$ E: $79 \%$
Se recomienda tratamiento de candidiasis esofágica con fluconazol oral como primera línea
Candidiasis invasora en paciente neutropénico
Anaissie, (1996) ${ }^{50}$
Fluconazol 400 mg/día E: $77 \%$
Anfotericina B

$0,67 \mathrm{mg} / \mathrm{kg} / \mathrm{día}$

E: $48 \%$
Se recomienda fluconazol para tratamiento de enfermedad moderada-severa

En pacientes no críticos, sin uso previo de azoles y sin alta incidencia de Candida no albicans, el uso de fluconazol es una buena alternativa

En pacientes sin neutropenia y sin inmunodeficiencia marcada, fluconazol y anfotericina B no tienen diferencias significativas en al tratamiento efectivo de candidemia

En pacientes neutropénicos, fluconazol demostró ser superior a anfotericina B para tratamiento de candidiasis invasora. 
Tabla 2. Estudios clínicos sobre el tratamiento de infecciones fúngicas con azoles (continuación)

\begin{tabular}{|c|c|c|c|c|}
\hline Escenario & Autor, año, referencia & Azol & Comparador & Recomendación \\
\hline \multicolumn{5}{|l|}{ Aspergilosis } \\
\hline \multirow[t]{2}{*}{ Invasora } & Herbrecht $(2002)^{42}$ & $\begin{array}{l}\text { Voriconazol } \\
\text { E: } 52,8 \%\end{array}$ & $\begin{array}{l}\text { Anfotericina B } \\
\text { E: } 31,6 \%\end{array}$ & $\begin{array}{l}\text { En pacientes con aspergilosis invasora } \\
\text { el tratamiento inicial con voriconazol } \\
\text { condujo a mejores respuestas y } \\
\text { mejoró la supervivencia }\end{array}$ \\
\hline & Maertens $(2015)^{51}$ & $\begin{array}{l}\text { Isavuconazol } 200 \text { mg IV } \\
\text { tres veces al día en los días } \\
1 \text { y } 2 \text {; luego una vez al día } \\
\text { IV u oral }\end{array}$ & $\begin{array}{l}\text { Voriconazol } \\
6 \mathrm{mg} / \mathrm{kg} \text { IV dos veces al día, el día 1; } \\
4 \mathrm{mg} / \mathrm{kg} \text { IV dos veces al día el día 2; } \\
\text { luego } 4 \mathrm{mg} / \mathrm{kg} \text { IV dos veces al día u } \\
200 \mathrm{mg} \text { oral dos veces día, desde el } \\
\text { día } 3 \text { en adelante }\end{array}$ & $\begin{array}{l}\text { No inferioridad para mortalidad, por } \\
\text { todas las causas, de isavuconazol vs } \\
\text { voriconazol para el tratamiento de } \\
\text { infección fúngica invasora por mohos } \\
\text { como Al }\end{array}$ \\
\hline $\begin{array}{l}\text { Histoplasmosis } \\
\text { diseminada en VIH }\end{array}$ & Wheat ${ }^{43}$ & $\begin{array}{l}\text { Itraconazol } 300 \mathrm{mg} 3 \text { días } \\
\text { seguido de } 200 \mathrm{mg} 12 \\
\text { semanas } \\
\text { E: } 85 \%\end{array}$ & & $\begin{array}{l}\text { Itraconazol es una terapia de } \\
\text { inducción segura y efectiva para } \\
\text { histoplasmosis leve diseminada en } \\
\text { pacientes con SIDA. Se prefiere el uso } \\
\text { de suspensión para la administración } \\
\text { oral si está disponible }\end{array}$ \\
\hline
\end{tabular}

\section{Mucormicosis}

Tratamiento primario Marty $(2016)^{52}$

Tratamiento de rescate
Greenberg $(2005)^{53}$

\section{Isavuconazol $200 \mathrm{mg} \mathrm{IV} \mathrm{u}$} oral, 3 veces al día durante seis dosis:

$200 \mathrm{mg} / \mathrm{día}$ hasta

la resolución de la enfermedad, falla, o durante 180 días o más

Posaconazol $800 \mathrm{mg} / \mathrm{día}$ E: $79 \%$
Anfotericina B

Es una opción para individuos que han fallado con la terapia con anfotericina B o que tienen toxicidad limitante de la dosis
Eficacia similar de isavuconazol vs anfotericina B para mucormicosis invasora

\section{Paracoccidioidomicosis}

$\begin{array}{lll}\text { Borges }^{45} & \text { Itraconazol } & \text { Cotrimoxazol } \\ & \text { E: } 86,4 \% & \text { E: } 51,3 \%\end{array}$

Shikanai-Yasuda (2017) ${ }^{54} \quad$ Itraconazol 200 mg/día

Telles $(2007)^{55}$
Itraconazol $200 \mathrm{mg} / \mathrm{día}$ E: $94,4 \%$
Voriconazol $400 \mathrm{mg} / \mathrm{dí}$ E: $88,6 \%$

E: Porcentaje de eficacia clínica. Al: Aspergilosis invasiva. IV: Vía de administración intravenosa.

\section{Efectos adversos de azoles}

Los triazoles en la práctica clínica son medicamentos generalmente bien tolerados; sin embargo, poseen efectos adversos claramente reconocidos. Los más frecuentes reportados son gastrointestinales y síntomas constituciona- les que, en algunas cohortes, pueden alcanzar hasta $45 \%$. Los efectos adversos de mayor interés se encuentran en tres grupos: los relacionados con hepatotoxicidad, las alteraciones cardiovasculares (especialmente la prolongación del intervalo QT) y las reacciones de hipersensibilidad al medicamento. 
La hepatotoxicidad está relacionada con todos los azoles; puede observarse como una elevación intermedia o severa de bio-marcadores de injuria hepática, que puede configurar un patrón de hepatitis, colestasis y falla hepática fulminante. La inflamación hepática se ha documentado especialmente en el caso de la exposición a voriconazol; no está claro, sin embargo, la dosis ni el tiempo de exposición; lo más frecuente es observar fenómenos de toxicidad hepatocelular y también un patrón colestásico, con menor frecuencia ${ }^{56}$.

En todos los azoles por la vía enzimática del CYP3A4 se observa prolongación del intervalo QT, No obstante, se reportan problemas cardiovasculares en menos de $5 \%$ de los pacientes que reciben este fármaco; por lo general se observa en el contexto de pacientes críticamente enfermos o con patologías de base ${ }^{57}$.

En la Tabla 3 se resumen los efectos adversos más comunes de los azoles y su correspondiente nivel de frecuencia ${ }^{2,56,58)}$

\section{Interacciones farmacológicas}

En general, los azoles tienen las mismas contraindicaciones e interacciones.
No se recomienda su prescripción con medicamentos que usen sustratos del CYP3A4, en especial con los que producen prolongación del $\mathrm{QT}^{57} \mathrm{e}$ igualmente, con medicamentos inhibidores de la 3-hidroxi-3-metil-glutaril-CoA reductasa ${ }^{47}$.

Algunos como posaconazol están contraindicados con el uso de inhibidores de la calcineurina como tacrolimus y sirolimus, porque aumentan sus concentraciones en sangre; este efecto también ocurre sobre las concentraciones sanguíneas de ciclosporina ${ }^{62}$.

Los azoles interactúan con agentes anti-tuberculosos como rifampicina, especialmente voriconazol ${ }^{63}$ e itraconazol, reduciéndose las concentraciones de este último antifúngico incluso hasta en $50 \%$, lo que plantea un dilema importante en el tratamiento de co-infecciones como histoplasmosis y tuberculosis ${ }^{64}$.

Los anti-retrovirales son otro grupo de medicamentos que tienen múltiples interacciones con los azoles. Algunas recomendaciones son: posaconazol está contraindicado como tratamiento combinado con efavirenz o fosamprenavir; altas dosis de itraconazol no están recomendadas en pacientes que reciben inhibidor de proteasas; voriconazol no debe utilizarse con ritonavir como potenciador del inhibidor de proteasas ${ }^{58}$.

\begin{tabular}{|c|c|c|c|c|c|}
\hline & Fluconazol (58) & Itraconazol (2) & Voriconazol* (59) & Isavuconazol (60) & 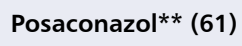 \\
\hline \multicolumn{6}{|l|}{ Hepático } \\
\hline Elevación de transaminasas & ++ & ++ & ++ & ++ & ++ \\
\hline Colestasia & + & ++ & + & + & + \\
\hline \multicolumn{6}{|l|}{ Gastrointestinal } \\
\hline Diarrea & ++ & ++ & ++ & +++ & ${ }^{*}+++$ \\
\hline Náuseas & ++ & +++ & ++ & +++ & ${ }^{*}+++$ \\
\hline Vómitos & ++ & ++ & ++ & +++ & ${ }^{*}+++$ \\
\hline Dolor abdominal & + & ++ & ++ & +++ & - \\
\hline \multicolumn{6}{|l|}{ Renal } \\
\hline Insuficiencia renal & + & $+/-$ & $+/-$ & +++ & $+/-$ \\
\hline \multicolumn{6}{|l|}{ Cutáneo } \\
\hline Exantema & ++ & ++ & ++ & ++ & - \\
\hline Alopecia & $+/-$ & - & - & $+/-$ & - \\
\hline \multicolumn{6}{|l|}{ Otros } \\
\hline Cardiovasculares & $+/-$ & $+/-$ & $+/-$ & ++ & $+/-$ \\
\hline Visuales & - & - & +++ & - & - \\
\hline Cefalea & ++ & +++ & ++ & + & +++ \\
\hline Fiebre & $+/-$ & $+/-$ & ++ & + & +++ \\
\hline Hematológicos & - & - & - & + & +++ \\
\hline Electrolitos & + & + & + & +++ & +++ \\
\hline
\end{tabular}




\section{Resistencia a anti-fúngicos azoles}

Los mecanismos de resistencia a azoles que se han descrito son dos:

El primero corresponde a la modificación de la diana, en el que cambios en la cantidad o calidad de la $14 \alpha$-desmetilasa u otras enzimas implicadas en la síntesis de ergosterol hacen que algunas especies requieran una mayor concentración de azoles para inhibir la síntesis de, por lo menos, $50 \%$ de ergosterol ${ }^{65}$. Las alteraciones enzimáticas más frecuentemente descritas se encuentran en el producto del gen CYP51/ERG11, en el que la mutación más caracterizada es la sustitución de arginina por lisina en el aminoácido $467^{66}$. Existen cepas de hongos que incrementan la síntesis de ergosterol, en relación con un elevado contenido microsomal de citocromo P450, como mecanismo de resistencia, lo que es común en el caso de $C$. glabrata.

El segundo mecanismo de resistencia es el eflujo activo. En éste, los hongos blanco de acción de los azoles, poseen proteínas pertenecientes a la superfamilia de proteínas de casete de unión de ATP (ABC), como CDR1 y CDR2, y de la superfamilia facilitadora principal (MFS), como MDR1, que se encargan de la expulsión de las moléculas de azoles ${ }^{67,68}$.

\section{Conclusiones}

Los triazoles: fluconazol, itraconazol, voriconazol, posaconazol e isavuconazol, son antimicóticos con una buena efectividad clínica para el tratamiento de la EFI, como es el caso de fluconazol para candidiasis y criptococosis, voriconazol en aspergilosis, itraconazol en histoplasmosis, posaconazol para el tratamiento de rescate de mucormicosis e isavuconazol para aspergilosis y mucormicosis. Los azoles tienen mejor perfil de seguridad que anfotericina $\mathrm{B}$; sin embargo, ocasionan varios efectos adversos como hepatotoxicidad y prolongación del intervalo QT. Además, tienen muchas interacciones farmacológicas, por lo que deben ser usados en contextos específicos y con precaución.

\section{Referencias bibliográficas}

1.- Peman J, Salavert M. General epidemiology of invasive fungal disease. Enferm Infecc Microbiol Clin 2012; 30: 90-8. doi: 10.1016/j. eimc.2011.09.004.

2.- Nucci M, Queiroz-Telles F, Tobon A M, Restrepo A y Colombo A L. Epidemiology of opportunistic fungal infections in Latin America. Clin Infect Dis 2010; 51: 561-70. doi: $10.1086 / 655683$.

3.- Allen D, Wilson D, Drew R y Perfect J. Azole antifungals: 35 years of invasive fungal infection management. Expert Rev Anti Infect Ther 2015; 13: 787-98. doi: 10.1586/14787210.2015.1032939.

4.- Pfaller M A, Diekema D J. Epidemiology of invasive mycoses in North America. Crit Rev Microbiol 2010; 36: 1-53. doi: 10.3109/10408410903241444

5.- Neofytos D, Horn D, Anaissie E, Steinbach W, Olyaei A, Fishman J, et al. Epidemiology and outcome of invasive fungal infection in adult hematopoietic stem cell transplant recipients: analysis of Multicenter Prospective Antifungal Therapy (PATH) Alliance registry. Clin Infect Dis 2009; 48: 265-73. doi: 10.1086/595846.

6.- Sipsas N V, Gamaletsou M N, Anastasopoulou A, Kontoyiannis D P. Therapy of mucormycosis. J Fungi (Basel) 2018; 4: 90. doi: 10.3390/jof4030090.

7.- Morales-López S E, Parra-Giraldo C M, Ceballos-Garzón A, Martínez H P, Rodríguez G J, Álvarez-Moreno C A, et al. Invasive infections with multidrug-resistant yeast Candida auris, Colombia. Emerg Infect Dis 2017; 23: 162-4. doi: 10.3201/eid2301.161497.

8.- Leonardelli F, Macedo D, Dudiuk C, Cabeza M S, Gamarra S, García-Effron G. Aspergillus fumigatus intrinsic fluconazole resistance is due to the naturally occurring T301I substitution in Cyp51Ap. Antimicrob Agents Chemother 2016; 60: 5420-6. doi: 10.1128/AAC.00905-16.

9.- Sheehan D J, Hitchcock C A, Sibley C M. Current and emerging azole antifungal agents. Clin Microbiol Rev 1999; 12: 40-79. PMID: 9880474.

10.- Shadomy S. In vitro antifungal activity of clotrimazole (Bay b 5097). Infect Immun 1971; 4: 143-8. PMID: 4949484.

11.- FDA Drug Safety Communication: FDA limits usage of Nizoral (ketoconazole) oral tablets due to potentially fatal liver injury and risk of drug interactions and adrenal gland problems. Available from: https://www.fda.gov/drugs/ drug-safety-and-availability/fda-drug-safetycommunication-fda-limits-usage-nizoralketoconazole-oral-tablets-due-potentially.

12.- European Medicines Agency recommends suspension of marketing authorisations for oral ketoconazole. Availabre from: https://www. ema.europa.eu/en/news/european-medicinesagency-recommends-suspension-marketingauthorisations-oral-ketoconazole.

13.- Yan J Y, Nie X L, Tao Q M, Zhan S Y, Zhang Y D. Ketoconazole associated hepatotoxicity: a systematic review and meta-analysis. Biomed Environ Sci 2013; 26: 605-10. doi: 10.3967/0895-3988.2013.07.013.

14.- Ministerio de Salud Panama. Resolución retiro ketoconazol. Available from:. http:// www.minsa.gob.pa/sites/default/files/alertas/ resolucion_de_ketoconazol.pdf.

15.- Maertens JA. History of the development of azole derivatives. Clin Microbiol Infect 2004; 10 Suppl 1: 1-10. doi: 10.1111/j.14709465.2004.00841.x.

16.- De Sarro A, La Camera E, Fera M T. New and investigational triazole agents for the treatment of invasive fungal infections. J Chemother 2008; 20: 661-71. doi: 10.1179/ joc.2008.20.6.661.

17.- Perfect J R, Marr K A, Walsh T J, Greenberg R N, DuPont B, de la Torre-Cisneros J, et al. Voriconazole treatment for lesscommon, emerging, or refractory fungal infections. Clin Infect Dis 2003; 36: 1122-31. doi: 10.1086/374557.

18.- Shirley M, Scott L J. Isavuconazole: A review in invasive aspergillosis and mucormycosis. Drugs 2016; 76: 1647-57. doi: 10.1007/s40265016-0652-6.

19.- Catalán M, Montejo J C. Antifúngicos sistémicos. Farmacodinamia y farmacocinetica. 2006: 39-49. http://www.reviberoammicol. com/2006-23/039049.pdf.

20.- Zonios D I, Bennett J E. Update on azole antifungals. Semin Respir Crit Care Med 2008; 29: 198-210. doi: 10.1055/s-2008-1063858.

21.- Ghanoumm M A, Rice L B. Antifungal agents: mode of action, mechanisms of resistance, and correlation of these mechanisms with bacterial 
resistance. Clin Microbiol Rev 1999; 12: 50117. PMID: 10515900.

22. Sanati H, Belanger P, Fratti R, Ghannoum M A new triazole, voriconazole (UK-109,496), blocks sterol biosynthesis in Candida albicans and Candida krusei. Antimicrob Agents Chemother 1997; 41: 2492-6. PMID: 9371355.

23.- Bossche H V, Marichal P, Le Jeune L, Coene M C, Gorrens J, Cools W. Effects of itraconazole on cytochrome P-450-dependent sterol 14?? demethylation and reduction of 3-ketosteroids in Cryptococcus neoformans. Antimicrob Agents Chemother 1993; 37: 2101-5. doi: 10.1128/aac.37.10.2101.

24.- Wang E J, Lew K, Casciano C N, Clement $\mathrm{R}$ P, Johnson W W. Interaction of common azole antifungals with $\mathrm{P}$ glycoprotein. Antimicrob Agents Chemother 2002; 46: 160-5. doi: 10.1128/aac.46.1.160-165.2002.

25.- Bruggemann R J, Alffenaar J W, Blijlevens N M, Billaud E M, Kosterink J G, Verweij P E, et al. Clinical relevance of the pharmacokinetic interactions of azole antifungal drugs with other coadministered agents. Clin Infect Dis 2009; 48: 1441-58. doi: 10.1086/598327.

26.- Sinnollareddy M, Peake S L, Roberts M S, Lipman J, Roberts J A. Using pharmacokinetics and pharmacodynamics to optimise dosing of antifungal agents in critically ill patients: a systematic review. Int J Antimicrob Agents 2012; 39: 1-10. doi: 10.1016/j. ijantimicag.2011.07.013.

27.- Kim S H, Kwon J C, Park C, Han S, Yim D S, Choi J K, et al. Therapeutic drug monitoring and safety of intravenous voriconazole formulated with sulfobutylether betacyclodextrin in haematological patients with renal impairment. Mycoses 2016; 59: 644-51. doi: 10.1111/myc. 12517 .

28.- Lass-Flörl C. Triazole antifungal agents in invasive fungal infections: a comparative review. Drugs 2011; 71: 2405-19. doi: 10.2165/11596540-000000000-00000.

29.- Azanza J R, García-Quetglas E, Sadaba B. Pharmacology of azoles. Rev Iberoam Micol 2007; 24: 223-7. doi: 10.1016/s11301406(07)70047-5.

30.- Willems L, van der Geest R, de Beule K. Itraconazole oral solution and intravenous formulations: a review of pharmacokinetics and pharmacodynamics. J Clin Pharm Ther 2001; 26: 159-69. doi: 10.1046/j.13652710.2001.00338.x.

31.- Moore J N, Healy J R, Kraft W K. Pharmacologic and clinical evaluation of posaconazole. Expert Rev Clin Pharmacol 2015; 8: 321-34. doi: $10.1586 / 17512433.2015 .1034689$.

32.- Leveque D, Nivoix Y, Jehl F, Herbrecht R. Clinical pharmacokinetics of voriconazole. Int J Antimicrob Agents 2006; 27: 274-84. doi: 10.1016/j.ijantimicag.2006.01.003.
33.- Jenks J D, Salzer H J, Prattes J, Krause R, Buchheidt D, Hoenigl M. Spotlight on isavuconazole in the treatment of invasive aspergillosis and mucormycosis: design, development, and place in therapy. Drug Des Devel Ther 2018; 12: 1033-44. doi: 10.2147/ DDDT.S145545.

34.- Ledoux M P, Denis J, Nivoix Y, Herbrecht R. Isavuconazole: A new broad-spectrum azole. Part 2: pharmacokinetics and clinical activity. J Mycol Med 2018; 28: 15-22. doi: 10.1016/j. mycmed.2018.02.002.

35.- del Palacio A, Villar J, Alhambra A. Epidemiología de las candidiasis invasoras en población pediátrica y adulta. Rev Iberoam Micol 2009; 26: 2-7. http://www. reviberoammicol.com/2009-26/002007.pdf.

36.- Cornely O A, Arikan-Akdagli S, Dannaoui E, Groll A H, Lagrou K, Chakrabarti A, et al. ESCMID and ECMM joint clinical guidelines for the diagnosis and management of mucormycosis 2013. Clin Microbiol Infect 2014; 20 Suppl 3: 5-26. doi: 10.1111/14690691.12371.

37.- Begg E J, Barclay M L, Kirkpatrick C M. The therapeutic monitoring of antimicrobial agents. Br J Clin Pharmacol 2001; 52 Suppl 1: 35S-43S. doi: 10.1046/j.13652125.2001.0520s1035.x.

38.- Hope W W, Billaud E M, Lestner J, Denning D $\mathrm{W}$. Therapeutic drug monitoring for triazoles. Curr Opin Infect Dis 2008; 21: 580-6. doi: 10.1097/QCO.0b013e3283184611.

39.- Day J N, Chau T T H, Wolbers M, Mai P P, Dung N T, Mai N H, et al. Combination antifungal therapy for cryptococcal meningitis. N Engl J Med 2013; 368: 1291-302. doi: 10.1056/NEJMoa1110404.

40.- Bozzette S A, Larsen R A, Chiu J, Leal M A, Jacobsen J, Rothman P, et al. A placebocontrolled trial of maintenance therapy with fluconazole after treatment of cryptococcal meningitis in the acquired immunodeficiency syndrome. California Collaborative Treatment Group. N Engl J Med 1991; 324: 580-4. doi: 10.1056/NEJM199102283240902.

41.- Villanueva A, Gotuzzo E, Arathoon E G, Noriega L M, Kartsonis N A, Lupinacci R $\mathrm{J}$, et al. A randomized double-blind study of caspofungin versus fluconazole for the treatment of esophageal candidiasis. Am J Med 2002; 113: 294-9. doi: 10.1016/s00029343(02)01191-9.

42.- Herbrecht R, Denning D W, Patterson T F, Bennett J E, Greene R E, Oestmann J W, et al. Voriconazole versus amphotericin $B$ for primary therapy of invasive aspergillosis. N Engl J Med 2002; 347: 408-15. doi: 10.1056/ NEJMoa020191.

43.- Wheat J, Hafner R, Korzun A H, Limjoco M T, Spencer P, Larsen R A, et al. Itraconazole treatment of disseminated histoplasmosis in patients with the acquired immunodeficiency syndrome. AIDS Clinical Trial Group. Am J Med 1995; 98: 336-42. doi: 10.1016/s00029343(99)80311-8.

44.- Donnelley M A, Zhu E S, Thompson G $\mathrm{R}$, 3rd. Isavuconazole in the treatment of invasive aspergillosis and mucormycosis infections. Infect Drug Resist 2016; 9: 79-86. doi: 10.2147/IDR.S81416.

45.- Borges S R, Silva G M, Chambela Mda C, Oliveira Rde V, Costa R L, Wanke B, et al. Itraconazole vs trimethoprim-sulfamethoxazole: A comparative cohort study of 200 patients with paracoccidioidomycosis. Med Mycol 2014; 52: 303-10. doi: 10.1093/mmy/myt012.

46.- Perfect J R, Dismukes W E, Dromer F, Goldman D L, Graybill JR, Hamill R J, et al. Clinical Practice Guidelines for the Management of Cryptococcal Disease: 2010 Update by the Infectious Diseases Society of America. Clin Infect Dis 2010; 50 (3): 291-322. doi: $10.1086 / 649858$.

47.- Reboli A C, Rotstein C, Pappas P G, Chapman S W, Kett D H, Kumar D, et al. Anidulafungin versus fluconazole for invasive candidiasis. $\mathrm{N}$ Engl J Med 2007; 356: 2472-82. doi: 10.1056/ NEJMoa066906.

48.- Pons V, Greenspan D, Debruin M. Therapy for oropharyngeal candidiasis in HIV-infected patients: a randomized, prospective multicenter study of oral fluconazole versus clotrimazole troches. The Multicenter Study Group. J Acquir Immune Defic Syndr 1993; 6: 1311-6. PMID: 8254467.

49.- Brandes R P, Kreuzer J. Vascular NADPH oxidases: molecular mechanisms of activation. Cardiovasc Res 2005; 65: 16-27. doi: 10.1016/j. cardiores.2004.08.007.

50.- Anaissie EJ, Darouiche RO, Abi-Said D, Uzun O, Mera J, Gentry LO, et al. Management of invasive candidal infections: results of a prospective, randomized, multicenter study of fluconazole versus amphotericin B and review of the literature. Clin Infect Dis 1996; 23: 964 72. doi: 10.1093/clinids/23.5.964.

51.- Maertens J A, Raad I I, Marr K A, Patterson T F, Kontoyiannis D P, Cornely O A, et al. Isavuconazole versus voriconazole for primary treatment of invasive mould disease caused by Aspergillus and other filamentous fungi (SECURE): a phase 3, randomised-controlled, non-inferiority trial. Lancet 2016; 387: 760-9. doi: 10.1016/S0140-6736(15)01159-9.

52.- Marty F M, Ostrosky-Zeichner L, Cornely O A, Mullane K M, Perfect J R, Thompson $\mathrm{G} \mathrm{R}$, 3rd, et al. Isavuconazole treatment for mucormycosis: a single-arm open-label trial and case-control analysis. Lancet Infect Dis 2016; 16: 828-37. doi: 10.1016/S14733099(16)00071-2.

53.- Greenberg R N, Mullane K, van Burik J A, Raad I, Abzug M J, Anstead G, et 
al. Posaconazole as salvage therapy for zygomycosis. Antimicrob Agents Chemother 2006; 50: 126-33. doi: 10.1128/AAC.50.1.126133.2006 .

54.- Shikanai-Yasuda M A, Mendes R P, Colombo A L, Queiroz-Telles F, Kono A S G, Paniago A M M, et al. Brazilian guidelines for the clinical management of paracoccidioidomycosis. Rev Soc Bras Med Trop 2017; 50: 715-40. doi: 10.1590/00378682-0230-2017.

55.- Queiroz-Telles F, Goldani L Z, Schlamm H T, Goodrich J M, Espinel-Ingroff A, ShikanaiYasuda M A. An open-label comparative pilot study of oral voriconazole and itraconazole for long-term treatment of paracoccidioidomycosis. Clin Infect Dis 2007; 45: 1462-9. doi: $10.1086 / 522973$.

56.- Kyriakidis I, Tragiannidis A, Munchen S, Groll A H. Clinical hepatotoxicity associated with antifungal agents. Expert Opin Drug Saf 2017; 16: 149-65. doi: 10.1080/14740338.2017.1270264.

57.- Salem M, Reichlin T, Fasel D, LeuppiTaegtmeyer A. Torsade de pointes and systemic azole antifungal agents: Analysis of global spontaneous safety reports. Glob Cardiol Sci Pract 2017; 2017: 11. doi: 10.21542/ gcsp.2017.11.

58.- Vadlapatla R K, Patel M, Paturi D K, Pal
D, Mitra A K. Clinically relevant drugdrug interactions between antiretrovirals and antifungals. Expert Opin Drug Metab Toxicol 2014; 10: 561-80. doi: 10.1517/17425255.2014.883379.

59.- Voriconazole. FDA. Available from: https:// www.accessdata.fda.gov/drugsatfda_docs/ label/2015/021266s038,021267s047, 021630s028lbl.pdf.

60.- Isavuconazole. FDA. Available from: https:// www.accessdata.fda.gov/drugsatfda_docs/ label/2015/207500Orig1s000lbl.pdf.

61.- Greer ND. Posaconazole (Noxafil): a new triazole antifungal agent. Proc (Bayl Univ Med Cent) 2007; 20: 188-96. doi: 10.1080/08998280.2007.11928283.

62.- Cendejas-Bueno E, Cuenca-Estrella M, Gómez-López A. Indicaciones clínicas de la monitorización de azoles de uso sistémico. Hacia la optimización del tratamiento de la infección fúngica. Rev Esp Quimioter 2014; 27: 01-16. https://seq.es/seq/0214-3429/27/1/ cendejas.pdf.

63.- Geist M J, Egerer G, Burhenne J, Riedel K D, Mikus G. Induction of voriconazole metabolism by rifampin in a patient with acute myeloid leukemia: importance of interdisciplinary communication to prevent treatment errors with complex medications. Antimicrob Agents Chemother 2007; 51: 3455-6. DOI: 10.1128/
AAC.00579-07

64.- Agudelo C A, Restrepo C A, Molina D A, Tobon A M, Kauffman C A, Murillo C, et al. Tuberculosis and histoplasmosis co-infection in AIDS patients. Am J Trop Med Hyg 2012; 87 : 1094-8. doi: 10.4269/ajtmh.2012.12-0292.

65.- Orozco A S, Higginbotham L M, Hitchcock C A, Parkinson T, Falconer D, Ibrahim AS, et al. Mechanism of fluconazole resistance in Candida krusei. Antimicrob Agents Chemother 1998; 42: 2645-9. PMID: 9756770.

66.- López-Ávila K, Dzul-Rosado K R, LugoCaballero C, Arias-León J J, Zavala-Castro J E. Mecanismos de resistencia antifúngica de los azoles en Candida albicans. Una revisión. Revista Biomédica 2016; 27: 127-36. https:// www.medigraphic.com/pdfs/revbio/bio-2016/ bio163e.pdf.

67.- vanden Bossche H, Marichal P, Odds F C, Le Jeune L, Coene M C. Characterization of an azole-resistant Candida glabrata isolate. Antimicrob Agents Chemother 1992; 36: 2602 10. doi: 10.1128/aac.36.12.2602.

68.- Fuentes M, Hermosilla G, Alburquenque C, Falconer M A, Amaro J, Tapia C. Characterization of azole resistance mechanisms in Chilean clinical isolates of Candida albicans. Rev Chilena Infectol 2014; 31: 511-7. http://dx.doi.org/10.4067/S071610182014000500001 . 